\title{
Prolonged avoidance training exacerbates OCD-like behaviors in a rodent model
}

\author{
Freddyson J. Martínez-Rivera (1,3, Marcos J. Sánchez-Navarro', Carlos I. Huertas-Pérez', Benjamin D. Greenberg², \\ Steven A. Rasmussen² and Gregory J. Quirk
}

\begin{abstract}
Obsessive-compulsive disorder (OCD) is characterized by compulsive behaviors that often resemble avoidance of perceived danger. OCD can be treated with exposure-with-response prevention (ERP) therapy in which patients are exposed to triggers but are encouraged to refrain from compulsions, to extinguish compulsive responses. The compulsions of OCD are strengthened by many repeated exposures to triggers, but little is known about the effects of extended repetition of avoidance behaviors on extinction. Here we assessed the extent to which overtraining of active avoidance affects subsequent extinction-with-response prevention (Ext-RP) as a rodent model of ERP, in which rats are extinguished to triggers, while the avoidance option is prevented. Male rats conditioned for $8 \mathrm{~d}$ or $20 \mathrm{~d}$ produced similar avoidance behavior to a tone paired with a shock, however, the $20 \mathrm{~d}$ group showed a severe impairment of extinction during Ext-RP, as well as heightened anxiety. Furthermore, the majority of overtrained (20d) rats (75\%) exhibited persistent avoidance following Ext-RP. In the $8 \mathrm{~d}$ group, only a minority of rats (37\%) exhibited persistent avoidance, and this was associated with elevated activity (c-Fos) in the prelimbic cortex and nucleus accumbens. In the $20 \mathrm{~d}$ group, the minority of non-persistent rats (25\%) showed elevated activity in the insular-orbital cortex and paraventricular thalamus. Lastly, extending the duration of Ext-RP prevented the deleterious effects of overtraining on extinction and avoidance. These rodent findings suggest that repeated expression of compulsion-like behaviors biases individuals toward persistent avoidance and alters avoidance circuits, thereby reducing the effectiveness of current extinction-based therapies.
\end{abstract}

\section{Introduction}

Obsessive-compulsive disorder $(\mathrm{OCD})$ is a chronic illness characterized by compulsive urges resembling avoidance of perceived danger ${ }^{1}$. Behaviorally, OCD is treated with exposure-with-response prevention (ERP) therapy, in which patients are coached to refrain from carrying out compulsions in response to environmental triggers, with the intention of extinguishing those behaviors. Due to their repetitive nature, OCD compulsions have been

Correspondence: Freddyson J. Martínez-Rivera

(freddyson.martinez-rivera@mssm.edu)

${ }^{1}$ Departments of Psychiatry and Anatomy \& Neurobiology, School of Medicine, Medical Sciences Campus, University of Puerto Rico, San Juan, PR 00936, USA ${ }^{2}$ Department of Psychiatry and Human Behavior, Alpert Medical School of Brown University and Butler Hospital and the Providence VA Medical Center, Providence, RI 02906, USA

Full list of author information is available at the end of the article hypothesized to originate from prolonged and repetitive exposure to avoidance-associated triggers ${ }^{2,3}$. However, little is known about the effects of long-term, repetitive avoidance behavior on subsequent extinction. OCD patients show deficits in fear extinction ${ }^{4,5}$ and goal-directed behaviors $^{6}$, as well as an altered cortico-striatal system $^{4,6,7}$. Extinction impairments are also observed in patients suffering from other psychiatric disorders such as posttraumatic stress disorders (PTSD) ${ }^{8}$, but are not typically accompanied by obsessions ${ }^{9}$. Although ERP is effective ${ }^{10,11}$, compulsions persist for many patients despite treatment ${ }^{12,13}$. Therefore, there is a pressing need to understand how OCD-related circuits and decision-making processes may be altered by long-term repetitive compulsions.

We previously developed a rodent model of ERP therapy ${ }^{14}$ by modifying an active avoidance task in which rats 
pressing a bar for food can avoid a tone-signaled shock by stepping onto a nearby platform ${ }^{15}$. After conditioning, rats are given several days of extinction training with a barrier inserted to prevent access to the platform (extinction-with-response prevention, Ext-RP). However, the duration of avoidance training in our previous experiment (10 days) may not have been sufficient to have an impact on the development of OCD-like behavior in rats. Extensive conditioning of avoidance may resemble prolonged expression of compulsions, which are thought to be reinforced by the reduction in patients' stress and anxiety $^{16}$. Therefore, to better approximate the long-term expression of compulsions, we tested the effect of avoidance overtraining ( 20 days vs. 8 days) on Ext-RP. Following Ext-RP, the activity profile of these two groups were compared using c-Fos indexing to characterize the effects of avoidance overtraining on brain circuits signaling subsequent expression of avoidance.

\section{Materials and methods}

\section{Subjects and bar-press training}

A total of seventy-eight adult male Sprague-Dawley rats (300-380 g; Envigo Laboratories, Indianapolis, IN, USA) were used in this study. Rats were restricted to $18 \mathrm{~g} /$ day of standard laboratory chow and trained to press a bar for sucrose pellets on a variable interval schedule of reinforcement averaging $30 \mathrm{~s}$ (VI-30s). Rats were food trained until they reached a criterion of $\geq 10$ presses $/ \mathrm{min}$. All procedures were approved by the Institutional Animal Care and Use Committee of the University of Puerto Rico School of Medicine, and the Association for Assessment and Accreditation of Laboratory Animal Care (AAALAC).

\section{Platform-mediated avoidance and extinction-with- response prevention}

Platform-mediated avoidance (PMA) training was performed as previously described ${ }^{15,17}$. Briefly, rats were conditioned with a pure tone $(30 \mathrm{~s}, 4 \mathrm{kHz}, 75 \mathrm{~dB})$ coterminating with an electric shock delivered through a grid floor $(2 \mathrm{~s}, 0.4 \mathrm{~mA})$. Rats received nine tone-shock pairings each day for 8 or 20 days, with a variable inter-trial interval averaging $3 \mathrm{~min}$. An opaque acrylic square platform $(14.0 \mathrm{~cm}$ each side, $0.33 \mathrm{~cm}$ tall $)$ was placed in the opposite corner of the sucrose-delivering bar, to allow rats to avoid the shocks. The platform was present throughout bar-press training to reduce novelty. On either day 9 or day 21, rats received extinction-withresponse prevention (Ext-RP) training, in which 15 tones without shocks were delivered per day (Pavlovian extinction), over 4 or 10 days, while access to the platform was blocked with a Plexiglas barrier (Test 1) as previously described (Rodriguez-Romaguera et al. ${ }^{14}$ ). Following Ext$\mathrm{RP}$ training, the barrier was then removed to assess transfer of extinction learning to avoidance behavior.
Behavioral data (position (center of mass), freezing, pressing) was acquired using the Any-Maze tracking system (Stoelting Co).

\section{Persistence tests}

In the first post-Ext-RP test (Test 1 ), rats were given a single tone with the barrier removed and the time spent on the platform was used as index of persistent avoidance. One hour later, persistent animals were divided into two groups and given a single tone either without the barrier present (Test 2a) or with the barrier placed in the adjacent corner where it did not block entrance to the platform (Test 2b). The following day, persistent rats underwent an additional one-tone test in which both the barrier and the platform were removed (Test 3 ). Because animals exposed to either the presence or absence of the barrier at Test 2 showed no statistical differences at Test 3 (8d: barrier vs. no barrier: $p=0.96$; 20d: barrier vs. no barrier: $p=0.45$ ), they were combined in their respective main groups and compared (8d vs. 20d).

\section{Elevated plus maze}

To determine whether overtraining induced generalized anxiety, a subset of animals from $8 \mathrm{~d}$ and $20 \mathrm{~d}$ groups were tested in an elevated plus maze (EPM) 60 min following Test $1^{18}$. Subjects were placed in the center of the EPM facing an open arm, while the time spent in open vs. closed arms was recorded for $5 \mathrm{~min}$ using the Any-Maze tracking system (Stoelting Co).

\section{Immunohistochemistry}

c-Fos Immunohistochemistry (IHC) was performed as previously described ${ }^{15,19}$ with some modifications. One hour after Test 1 , a subset of rats was anesthetized with sodium pentobarbital $(450 \mathrm{mg} / \mathrm{Kg}$, i.p.) and transcardially perfused with $250 \mathrm{ml}$ of $0.9 \%$ saline followed by $500 \mathrm{ml}$ of cold fresh $4 \%$ paraformaldehyde (PFA) in $0.1 \mathrm{M}$ potassium phosphate buffer (PBS) at $\mathrm{pH}$ 7.4. Brains were removed and transferred to $30 \%$ sucrose in $0.1 \mathrm{M} \mathrm{PBS}$ for $48-72 \mathrm{~h}$, for cryoprotection. Using a cryostat (CM 1850; Leica), frozen sections $(40 \mu \mathrm{m})$ were prepared at the levels of prefrontal/orbital cortices, striatum, and amygdala/thalamus. Sections were initially washed (PBS) and blocked in a solution of $2 \%$ normal goat serum (NGS, Vector Laboratories) and 0.1\% tween (Tween-20, Sigma-Aldrich) in $0.1 \mathrm{M}$ PBS ( $\mathrm{pH} \mathrm{7.4)} \mathrm{for} 1 \mathrm{~h}$. The sections were then incubated overnight at room temperature (RT) with rabbit anti-c-Fos (Ab-5, Oncogene Science) at a concentration of 1:20,000. Sections were washed and then incubated for $2 \mathrm{~h}$ at $\mathrm{RT}$ in a solution of biotinylated goat anti-rabbit IgG (1:200; Vector Laboratories) and placed in a mixed avidin-biotin horseradish peroxidase complex solution (ABC Elite Kit, Vector Laboratories) for 90 min. Black immunoreactive nuclei labeled for $\mathrm{c}$-fos were visualized 
after $5 \mathrm{~min}$ of exposure to $\mathrm{DAB} /$ peroxidase substrate kit (Vector Laboratories). Sections were mounted on coatedgelatin slides, dehydrated and cover slipped. Counter sections were stained with Nissl and used to determine the anatomical boundaries of each structure analyzed.

c-Fos labeled neurons were automatically counted at 20x magnification with an Olympus microscope (Model BX51) equipped with a digital camera. We restricted our analysis to several areas previously implicated in conditioned fear or avoidance behavior ${ }^{14,15,17,20}$. Images were generated for subregions of prefrontal cortex $(+3.72$ to $2.52 \mathrm{AP})$, orbitofrontal cortex $(+4.20$ to $+3.00 \mathrm{AP})$, anterior agranular insular cortex $(+4.20$ to $+3.00 \mathrm{AP})$, dorsal striatum $(+2.16$ to $+1.20 \mathrm{AP})$, nucleus accumbens $(+2.04$ to $+1.20 \mathrm{AP})$, basal nucleus of the amygdala $(-2.28$ to $-3.12 \mathrm{AP})$, central nucleus of the amygdala $(-2.28$ to $-2.92 \mathrm{AP})$, paraventricular thalamus $(-2.28$ to -3.12 AP) ${ }^{21}$. Positive c-fos-like immunoreactivity showed brown-black staining distinct from the background. c-fos positive cells were automatically counted and averaged (blinded) for each hemisphere at 2-4 different rostrocaudal levels of each structure using Metamorph software 6.1 (Molecular Devices). The density of Fos labeling was calculated by dividing the number of positively labeled neurons by the total area of each region (counts $/ 0.1 \mathrm{~mm}^{2}$ ).

\section{Data analysis}

Student $t$-tests (two-sided), Fisher exact tests, and ANOVAs were performed to determine statistical differences in behavioral and immunohistochemistry experiments. For post hoc tests, we used Tukey (behavior) or Fisher LSD (c-Fos). Pearson correlation analyses were also performed. Data is presented as mean \pm standard error (SEM) and statistical significance was established as ${ }^{*} p<$ 0.05 (a minimum of subjects were used to achieve significance). All statistical analyses were performed using Statistica 6.0 (Statsoft ${ }^{\circledR}$ ) and Prism 6.0 (GraphPad Inc.) software's.

\section{Results}

\section{Avoidance behavior was similar following $8 \mathrm{~d}$ or $20 \mathrm{~d}$ of conditioning}

Food-deprived rats were separated (randomly) into two groups to receive either standard avoidance conditioning over 8 days (8d group) or extended avoidance conditioning over 20 days (20d group) (Fig. 1a-d). Rats learned to avoid a tone-signaled footshock by stepping onto a nearby platform ${ }^{15}$. Because the platform was positioned away from the food bar, avoidance incurred a cost of lost access to food. On the final day of conditioning, both $8 \mathrm{~d}$ and $20 \mathrm{~d}$ groups exhibited similar levels of avoidance expression, as indicated by the percent of the $30 \mathrm{~s}$ tone spent on the platform (8d: $89.1 \%, 20 \mathrm{~d}: 89.6 \%$ ), as well as the rate of bar pressing during the pre-tone interval (8d: 12 presses/min, 20d: 11 presses $/ \mathrm{min}$ ), and the amount of freezing to the tone (8d: $34.7 \%, 20 \mathrm{~d}$ : $34.6 \%$ ). Thus, following conditioning, $8 \mathrm{~d}$ and $20 \mathrm{~d}$ groups were behaviorally indistinguishable in this task.

\section{The $20 \mathrm{~d}$ group showed impaired extinction and persistent avoidance}

Following avoidance conditioning, rats received 4 days of extinction-with-response prevention (Ext-RP) training, in which tones were delivered without shocks in the presence of a Plexiglas barrier blocking access to the platform $^{14}$. During Ext-RP, repeated measures (RM) ANOVAs followed by Tukey post hoc analyses revealed that the 20d group was severely impaired in extinction of conditioned freezing as compared with the $8 \mathrm{~d}$ group $\left(F_{(1}\right.$, 76) $=9.10 ; p<0.01)$ (Fig. 2a). On day $4,20 \mathrm{~d}$ rats concluded Ext-RP training with significantly elevated levels of freezing compared to the $8 \mathrm{~d}$ group $\left(F_{(1,76)}=11.18 ; p<\right.$ $0.01)$. The $20 \mathrm{~d}$ group also showed elevated suppression of bar pressing $\left(\mathrm{F}_{(1,76)}=13.55 ; p<0.01\right)$ (Fig. $2 \mathrm{a}$; inset) and a reduced rate of spontaneous pressing during the pre-tone interval $\left(\mathrm{F}_{(1,76)}=20.33 ; p<0.01\right)$ (Fig. 2a; lower panel). Impaired extinction in $20 \mathrm{~d}$ rats suggests that the lack of access to the platform triggered a generalized fear/anxiety state that persisted throughout Ext-RP.

After Ext-RP, when the barrier was removed (Test 1), the 20d group spent significantly more time on the platform both prior to the tone $\left(t_{(76)}=3.23, p<0.01\right)$ and during the tone $\left(t_{(76)}=4.03, p<0.01\right)$ compared to the $8 \mathrm{~d}$ group (Fig. 2b). However, avoidance at Test 1 was significantly reduced compared to the last day of conditioning for both groups (both $p$ 's $<0.01 ; t$-tests). Similarly, the $20 \mathrm{~d}$ group showed a decreased rate of lever pressing both prior to the tone $\left(t_{(76)}=3.00, p<0.01\right)$ and during the tone $\left(t_{(76)}=2.86\right.$, $p<0.01$ ) (Fig. $2 \mathrm{~b}$ lower panel). While the freezing levels at Test 1 were equivalent for both groups (8d: 26\%, 20d: 25\%), freezing in the $8 \mathrm{~d}$ group was increased relative to the end of Ext-RP, likely due to a contextual renewal effect caused by removal of the barrier. A RM ANOVA confirmed these results within and between groups across these time points (pre-tone vs. tone) for all behavioral measures (avoidance, freezing, and presses; Tukey post hoc analyses, all $p$ 's $<0.05$ with one exception: $p=0.06$ ). Following Test 1 , a subset of rats were tested in the elevated plus maze (EPM), and the 20d group exhibited elevated anxiety as indicated by reduced time spent in the open arms $\left(t_{(32)}=2.12, p=0.04\right.$ ) (Fig. 2c).

Together, these results indicate that overtraining of active avoidance impairs subsequent extinction, predisposing rats to persistent avoidance. These effects are unlikely due to the $20 \mathrm{~d}$ group receiving more shocks than the $8 \mathrm{~d}$ group during conditioning, because the total number of shocks received (due to failed avoidance trials) did not significantly differ between groups (8d: 18.0; 20d: 
a
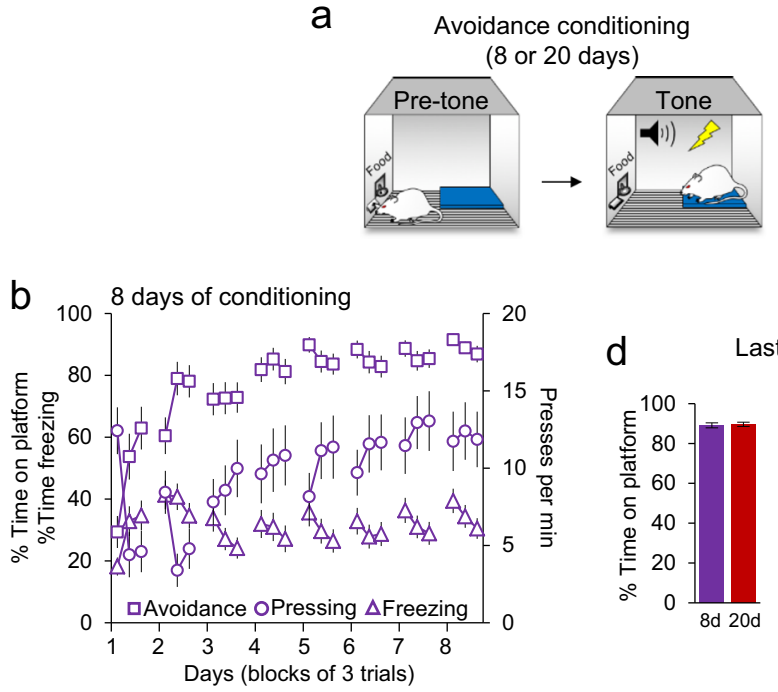

d Last day of conditioning
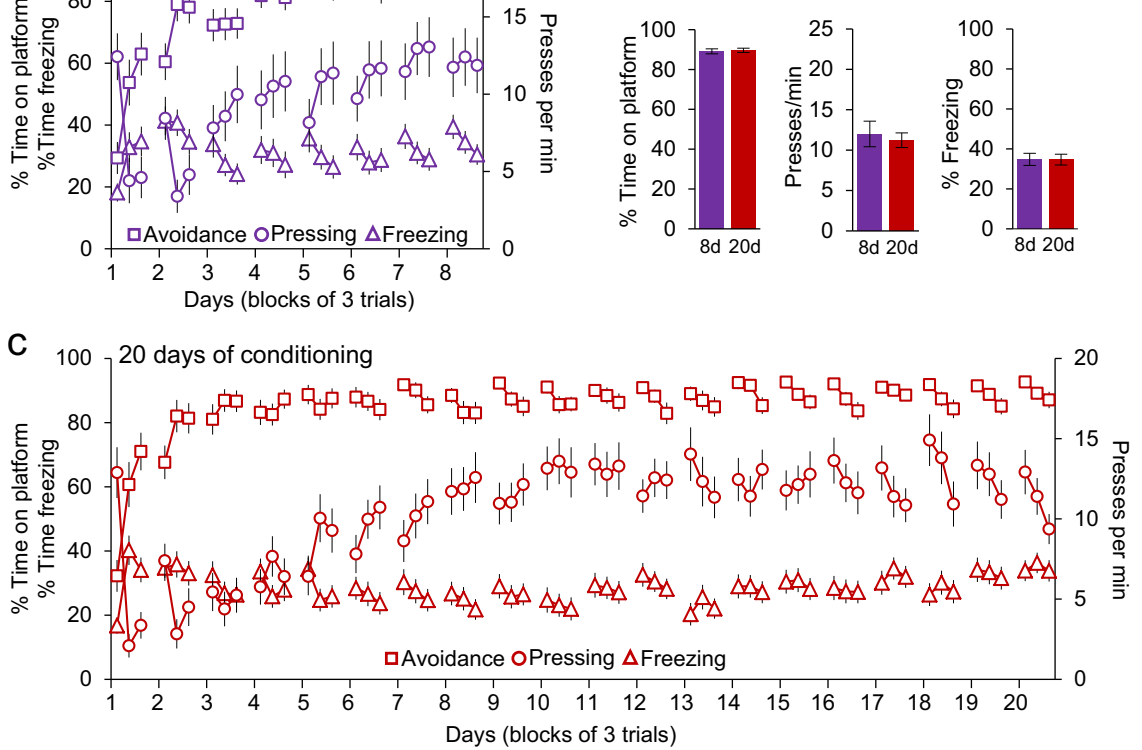

Fig. 1 Avoidance conditioning lasting $\mathbf{8 d}$ vs. 20d. a Rats pressing for sucrose pellets were trained to avoid a tone signaled foot-shock by stepping onto a nearby platform. b One group (8d) was conditioned over 8 days. As avoidance increased, freezing to the tone gradually decreased and bar pressing during the pre-tone interval gradually returned to preconditioning levels. c A different group (20d) was conditioned over 20 days. $\mathbf{d} 8 \mathrm{~d}$ and $20 \mathrm{~d}$ groups showed equivalent levels of avoidance, pressing, and freezing at the end of conditioning. $8 \mathrm{~d}$ group: $n=38 ; 20 \mathrm{~d}$ group: $n=40$. All data are shown of blocks of three trials as mean \pm SEM.

20.7; $t_{(76)}=1.65, p=0.11$ ). Because $8 \mathrm{~d}$ and $20 \mathrm{~d}$ groups showed equivalent avoidance, freezing, and pressing at the end of conditioned, this suggests that heightened anxiety in $20 \mathrm{~d}$ rats was induced by the loss of the avoidance option in the Ext-RP phase. A frequency distribution analysis of avoidance at Test 1 revealed a large subgroup of $20 \mathrm{~d}$ rats exhibiting $\geq 70 \%$ time on platform (Fig. 2d, e). We therefore used this cut-off value to define persistent avoidance behavior. Accordingly, 37\% $(n=14)$ of rats in the $8 \mathrm{~d}$ group were persistent $(\mathrm{P})$ whereas $63 \%(n=24)$ were non-persistent (NP). However, in the $20 \mathrm{~d}$ group, $75 \%(n=30)$ of rats were persistent, whereas $25 \%(n=10)$ were not. The ratio of persistence was significantly higher in the $20 \mathrm{~d}$ group (Fisher's exact test: $p<0.01$ ) (Fig. 2e; lower panels).

\section{Persistent avoidance in the $20 \mathrm{~d}$ group could be reduced with a contextual cue}

Subsets of rats from both groups that exhibited persistent avoidance were given two additional tests to assess the durability of their persistent avoidance (Fig. 3a, b). First, they were re-tested one hour after Test 1 with either no barrier present or with a barrier placed away from the platform (adjacent corner), so as not to block access to the platform. We reasoned that the presence of the barrier might act as a contextual occasion setter ${ }^{22,23}$ predictive of the absence of the shock because it first appeared when shocks were omitted.

Accordingly, we performed a two-way ANOVA followed by Tukey post hoc analysis to capture potential differences of $8 \mathrm{~d}$ and $20 \mathrm{~d}$ groups through Test 1 , Test $2 \mathrm{a}$ (no barrier), and Test $2 \mathrm{~b}$ (barrier). This analysis revealed a significant main effect of Test $\left(F_{(3,78)}=45.15, p<0.01\right)$, treatment group $\left(F_{(1,78)}=8.54, p<0.01\right)$ and interaction $\left(F_{(3,38)}=3.55, p=0.01\right)$, confirming that $8 \mathrm{~d}$ rats showed a reduction in avoidance from Test 1 to Test 2a (89-28\%, $p<0.01)$, whereas no significant reduction was observed in the $20 \mathrm{~d}$ group $\left(87-70 \%, p=0.37\right.$ ) (Fig. $\left.3 \mathrm{~b}_{1}\right)$. Placing the barrier away from the platform significantly reduced avoidance from Test 1 to Test $2 \mathrm{~b}$ in both groups 


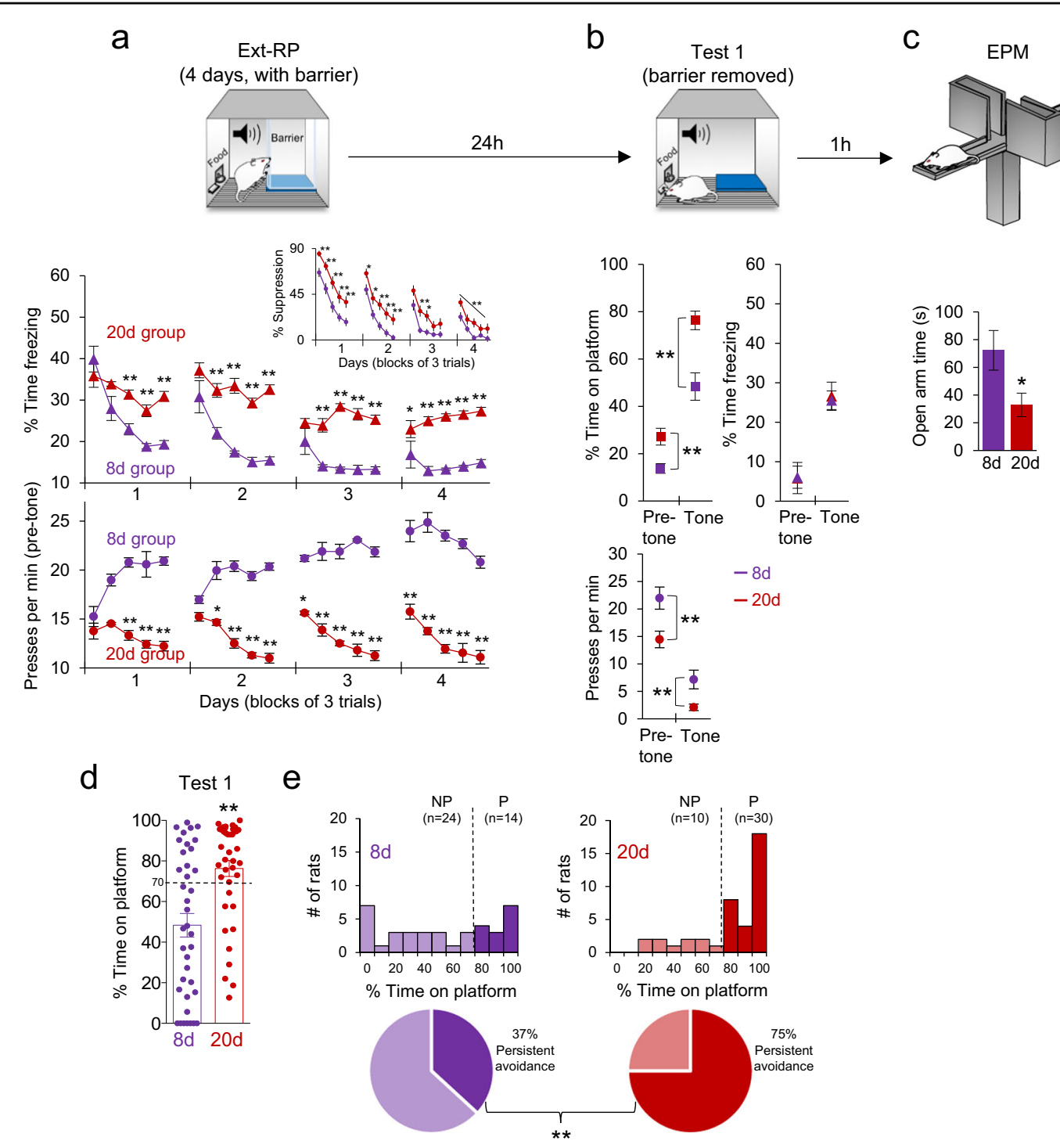

Fig. 2 Overtraining leads to impaired extinction (Ext-RP) and persistent avoidance. a Rats conditioned for $8 \mathrm{~d}$ or 20d were presented with tones in the absence of shock over 4 days, with a Plexiglas barrier inserted to block access to the platform (Ext-RP). Initially, both groups showed equivalent freezing, but extinction of freezing was impaired in the $20 \mathrm{~d}$ group, as was extinction of suppression of bar pressing (inset). Rats in the $20 \mathrm{~d}$ group were also impaired in their extinction of press suppression during the pre-tone period. $\mathbf{b}$ One day following Ext-RP training, rats were tested with the barrier removed (Test 1). The $20 \mathrm{~d}$ group showed excessive avoidance compared to the $8 \mathrm{~d}$ group, during both the pre-tone and tone periods, but there was no group difference in freezing. c The $20 \mathrm{~d}$ group also showed elevated anxiety, as indicated by reduced time spent in the open arms of an elevated plus maze (EPM). d, e Distribution of avoidance values (time on platform) during Test 1. Rats showing more than $70 \%$ time on platform were classified as persistent (P), whereas those showing less than 70\% were classified as non-persistence (NP). 8d group: $n=38 ; 20 \mathrm{~d}$ group: $n=40$. For EPM, 8d group: $n=20$; 20d group: $n=14$. Ext-RP data shown as blocks of three trials, whereas Test 1 data shown as single trial. All data are mean \pm SEM. ${ }^{*} p<0.05,{ }^{* *} p<0.01$.

(8d: $85-17 \%, p<0.01 ; 20$ d: $89-29 \%, p<0.01$ ) (Fig. $3 b_{2}$ ), suggesting that persistent avoidance in overtrained rats is sensitive to contextual occasion setters that signal safety $^{23-25}$.

In a final test, we removed both the barrier and the platform, and observed that rats in the $20 \mathrm{~d}$ group spent increased time in the corner where the platform had been located (RM ANOVA and Tukey post hoc) (Fig. 3c). Comparing Tests 1 and 3 revealed significant effects of Test $\left(F_{(1,11)}=8.20, p=0.01\right)$, group $\left(F_{(1,11)}=5.05\right.$, $p=0.04)$, and interaction $\left(\mathrm{F}_{(1,11)}=6.59, \quad p=0.02\right)$. Whereas the $8 \mathrm{~d}$ group showed a significant reduction in Test $3(86-39 \%, p<0.01)$, the $20 \mathrm{~d}$ group did not $(88-85 \%, p=0.99)$. The maintenance of avoidance in the $20 \mathrm{~d}$ group in the absence of the platform suggests that contextual cues (cage corner) were sufficient to drive 


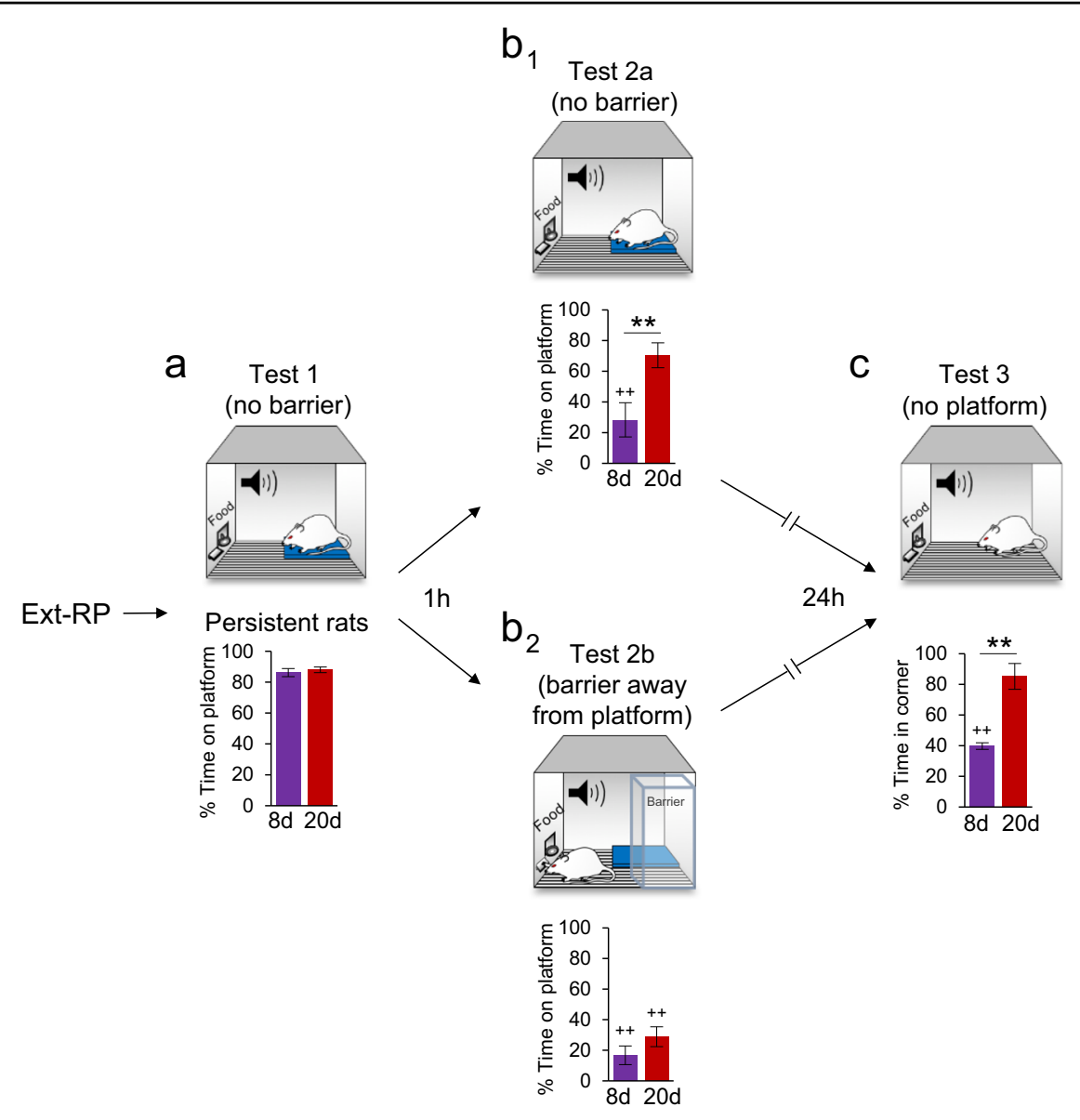

Fig. 3 Additional tests for avoidance persistence. a Rats classified as persistent avoiders in Test 1 were given additional tests for avoidance. $\mathbf{b}_{1}$ Simply re-testing the rats one hour later with the barrier still absent reduced avoidance in the $8 \mathrm{~d}$ group but not in the 20d group. $\mathbf{b}_{2}$ Re-testing a different subset of rats one hour later with the barrier placed in the adjacent corner (without blocking the platform) reduced avoidance in the 20d group, suggesting that the barrier acted as a contextual occasion setter signaling safety. c With both the barrier and platform removed, rats in the 20d (but not the $8 \mathrm{~d}$ ) group spent excessive time where the platform had been located, suggesting persistent avoidance. Test 1, 8d: $n=12 ; 20 \mathrm{~d}$ : $n=31$. Test 2 (no barrier), 8d: $n=5 ; 20 \mathrm{~d}: n=14$; Barrier (away from the platform), 8d: $n=7 ; 20 \mathrm{~d}: n=17$. Test 3 (no platform), 8d: $n=8 ; 20 \mathrm{~d}: n=5$. All data are shown as mean \pm SEM. ${ }^{* *} p<0.01$ between group differences; ${ }^{++} p<0.01$ within group differences as compared with their Test 1.

avoidance. Thus, persistent avoidance in overtrained animals is long-lasting and is modulated by contextual cues. Notably, no group differences in freezing levels were detected across the tests (ANOVA's and Tukey post hoc analyses, all $p$ 's $>0.05$ ).

\section{c-Fos revealed structures in the $8 \mathrm{~d}$ group signaling persistence, and structures in the $20 \mathrm{~d}$ group signaling non-persistence}

To examine differences in neuronal activity between $8 \mathrm{~d}$ and 20d groups, we obtained c-Fos expression profiles in a subset of rats sacrificed $60 \mathrm{~min}$ after Test 1 . We initially performed a two-way ANOVA (groups vs. brain regions) comparing c-Fos expression between $8 \mathrm{~d}$ and $20 \mathrm{~d}$ groups, however, no group differences were detected (main effect of group: $F_{(1,374)}=0.93, p=0.33$ ). We then proceeded to distinguish persistent (P) from non-persistent (NP) subgroups. Table 1 shows c-Fos values for all regions analyzed. A two-way ANOVA (groups $\times$ regions) detected a main effect of subgroup (NP vs P; $F_{(3,334)}=5.32, p<$ $0.01)$ and brain region $\left(\mathrm{F}_{(19,334)}=16.36, p<0.01\right)$, but not an interaction $\left(F_{(57,334)}=0.94, p=0.59\right)$, which indicates the substantial variability of $c$-fos levels among the 4 subgroups and 20 brain regions. Post hoc tests were performed to detect significant differences between subgroups in each region. In the $8 \mathrm{~d}$ group, the rats showing persistent avoidance exhibited increased activity in areas previously associated with avoidance in this task ${ }^{15,17}$ as compared to rats not showing persistence (one-way ANOVA, $F_{(3,17)}=24.67, p<0.01$, Tukey post hoc; Fig. $4 \mathrm{a}$ ). These areas include the nucleus accumbens core (NAccore, $p=0.03$ ) and a trend toward significance in prelimbic cortex (PL, $p=0.14$ ) (Fig. 4b). Expressed as a ratio of $\mathrm{P} / \mathrm{NP}$ groups, PL showed significantly more c-Fos 
Table 1 Brain structures signaling persistent and non-persistent avoidance behavior.

\begin{tabular}{|c|c|c|c|c|}
\hline Region & $8 d \mathrm{NP}$ & $8 d P$ & 20d NP & 20d P \\
\hline Prelimbic cortex (PL) & $12.5 \pm 2.1$ & $25.6 \pm 6.4^{\#+}$ & $11.3 \pm 1.5$ & $5.3 \pm 1.1$ \\
\hline Infralimbic cortex (IL) & $17.7 \pm 2.2$ & $27.6 \pm 6.2$ & $11.7 \pm 1.2$ & $9.0 \pm 2.1$ \\
\hline Medial orbital cortex (MO) & $25.4 \pm 7.3$ & $17.5 \pm 2.4$ & $37.0 \pm 14.0^{\#}$ & $22.6 \pm 8.8$ \\
\hline Ventral orbital cortex (VO) & $18.1 \pm 4.4$ & $24.1 \pm 8.8$ & $25.7 \pm 8.2$ & $15.4 \pm 5.6$ \\
\hline Lateral orbital cortex (LO), medial part & $54.5 \pm 13.5$ & $71.8 \pm 22.2^{\#}$ & $67.8 \pm 13.3$ & $68.3 \pm 26.9$ \\
\hline Lateral orbital cortex (LO), lateral part & $19.9 \pm 6.6$ & $19.6 \pm 5.6$ & $41.8 \pm 6.1^{*+}$ & $16.6 \pm 6.3$ \\
\hline Agranular insular cortex (Al), rostro-ventral part & $14.6 \pm 5.7$ & $12.0 \pm 4.6$ & $39.5 \pm 15.0^{*+}$ & $13.4 \pm 5.8$ \\
\hline Agranular insular cortex (Al), rostro-dorsal part & $20.1 \pm 5.1^{\#}$ & $5.8 \pm 2.2$ & $6.8 \pm 4.2$ & $2.5 \pm 1.6$ \\
\hline $\mathrm{Al} / \mathrm{LO}$ area & $17.3 \pm 5.6$ & $16.0 \pm 5.5$ & $40.0 \pm 3.3^{*+}$ & $15.0 \pm 5.6$ \\
\hline Nucleus accumbens core (NAc-core) & $19.0 \pm 3.0$ & $38.3 \pm 6.2^{*}$ & $23.4 \pm 6.2$ & $23.8 \pm 7.3$ \\
\hline Nucleus accumbens medial shell (NAc-mshell) & $8.4 \pm 1.4$ & $21.8 \pm 4.6^{\#}$ & $13.7 \pm 4.5$ & $12.7 \pm 3.8$ \\
\hline Nucleus accumbens lateral shell (NAc-Ishell) & $12.8 \pm 6.2$ & $11.6 \pm 6.1$ & $27.0 \pm 8.4$ & $21.4 \pm 11.6$ \\
\hline Dorsomedial striatum (DMS) & $2.2 \pm 1.6$ & $9.6 \pm 3.6$ & $9.2 \pm 3.3$ & $2.0 \pm 0.7$ \\
\hline Dorsolateral striatum (DLS) & $0.9 \pm 0.4$ & $0.9 \pm 0.5$ & $5.3 \pm 1.0$ & $2.2 \pm 0.6$ \\
\hline Basal amygdala (BA) & $17.3 \pm 2.8$ & $15.1 \pm 2.2$ & $14.4 \pm 2.8$ & $25.4 \pm 11.4$ \\
\hline Lateral amygdala (LA) & $10.5 \pm 1.4$ & $8.7 \pm 0.4$ & $10.2 \pm 0.6$ & $11.6 \pm 2.7$ \\
\hline Central amygdala, medial part (CeM) & $2.1 \pm 0.5$ & $6.1 \pm 2.1$ & $5.3 \pm 1.4$ & $4.2 \pm 2.0$ \\
\hline Central amygdala, lateral part (CeL) & $5.0 \pm 1.4$ & $7.9 \pm 1.6$ & $7.3 \pm 0.7$ & $6.1 \pm 2.4$ \\
\hline Mediodorsal thalamus (MD) & $4.9 \pm 1.4$ & $6.4 \pm 0.9$ & $8.4 \pm 2.9$ & $4.0 \pm 3.5$ \\
\hline Paraventricular thalamus (PVT) & $23.1 \pm 5.0$ & $39.2 \pm 7.0^{\#}$ & $48.9 \pm 5.9^{*+}$ & $19.9 \pm 4.8$ \\
\hline
\end{tabular}

NP vs. $P:{ }^{*} p<0.05 ;{ }^{*} p \leq 0.1$

$\mathrm{NP}$ vs. NP or $\mathrm{P}$ vs. $\mathrm{P}^{+} p<0.05$.

expression at $8 \mathrm{~d}$ compared to $20 \mathrm{~d}$ (8d: $153 \%$; 20d: $62 \%$; $\left.t_{(7)}=2.55, p=0.03\right)$. Surprisingly, however, the persistent rats in the $20 \mathrm{~d}$ group did not show increased activity in these areas (Fig. 4b). In fact, PL activity in 20d persistent rats was significantly lower than $8 \mathrm{~d}$ persistent rats $(p=$ 0.04; Fig. 4b). Indeed, none of the structures analyzed in the $20 \mathrm{~d}$ group showed increased activity in the persistent subgroup compared to the non-persistent subgroup (Table 1), suggesting that the persistent avoidance in the $20 \mathrm{~d}$ group was not mediated by the avoidance circuit previously described for this task.

In contrast to the $8 \mathrm{~d}$ group, the $20 \mathrm{~d}$ group showed elevated brain activity in the minority of rats showing non-persistence $(p<0.01$; Fig. $4 \mathrm{a})$. These structures were: the rostro-ventral portion of the agranular insular cortex (AI: $p=0.01$ ), the border of AI with the lateral portion of the lateral orbital cortex (AI/LO: $p=0.02)$, the lateral portion of the lateral orbital cortex (LO: $p=0.02$ ), and the paraventricular thalamus (PVT: $p<0.01$ ) (Fig. 4c). Thus, activation of cortico-striatal-thalamic areas was associated with the minority of rats in the $20 \mathrm{~d}$ group that were able to overcome persistence.

\section{The deleterious effects of overtraining could be prevented by extending Ext-RP}

Because the majority of 20d rats persisted in avoidance following Ext-RP, it could be said that they "failed" ExtRP. Increased avoidance at test might be expected, however, given the severe impairment in extinction in this group across the 4 days of Ext-RP (see Fig. 2a). We therefore asked if extending Ext-RP beyond 4 days could recover behavior in overtrained rats, by adding a new group in which rats were conditioned for $20 \mathrm{~d}$ and then given $10 \mathrm{~d}$ of Ext-RP training. The increase from $4 \mathrm{~d}$ to $10 \mathrm{~d}$ was selected to match the increase in conditioning from $8 \mathrm{~d}$ to $20 \mathrm{~d}(2.5 \times)$. To facilitate across-group comparisons, we also trained new sets of animals in the prior two conditions: regular training $(8 \mathrm{~d}-4 \mathrm{~d})$ and overtraining (20d-4d) (Fig. 5a).

As expected, we replicated our findings of impaired extinction $\left(F_{(2,50)}=4.70, \quad p=0.01\right)$ and persistent avoidance $\left(F_{(2,50)}=5.07, p<0.01\right)$ in $20 \mathrm{~d}-4 \mathrm{~d}$ rats compared with $8 \mathrm{~d}-4 \mathrm{~d}$ rats (all $p$ 's $<0.05$, one-way ANOVA and Tukey post hoc analyses; Fig. 5b). Importantly, the $20 \mathrm{~d}-10 \mathrm{~d}$ group resembled the $8 \mathrm{~d}-4 \mathrm{~d}$ group by 
a

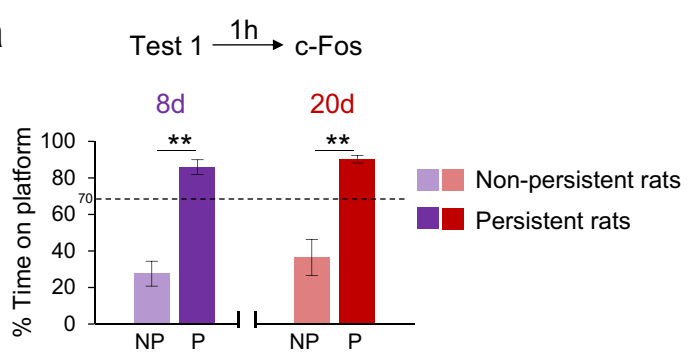

b

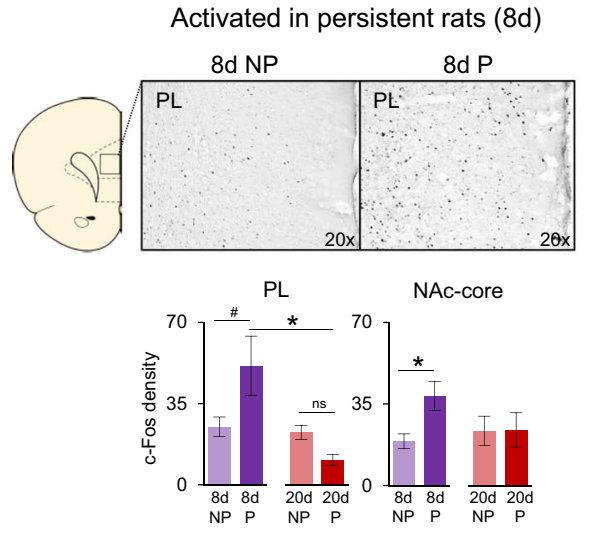

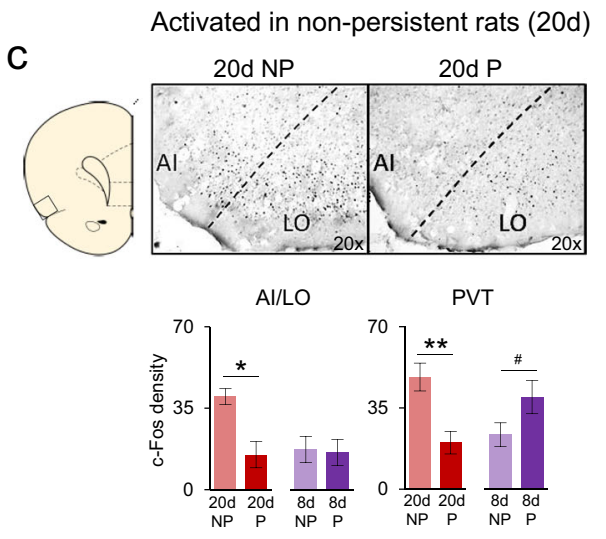

Fig. 4 c-Fos signaling of persistent vs. non-persistent avoidance. a Avoidance behavior at Test 1 in a subset of animals showing persistent (P) and non-persistent (NP) avoidance. One hour after Test 1, a subset of rats was processed for c-Fos immunocytochemistry. b Micrograph shows representative c-Fos labeling in prelimbic (PL) cortex for NP and P rats. 8d group data for c-Fos labeling in PL and nucleus accumbens-core (NAccore). The $8 \mathrm{~d}$ group showed elevated c-Fos in $\mathrm{P}$ rats compared to NP rats (or to $20 \mathrm{~d}$ P), suggesting signaling of persistence. $\mathbf{c}$ In contrast to $8 \mathrm{~d}$ rats, no structure in the $20 \mathrm{~d}$ group signaled persistence, but several structures showed elevated activity in non-persistent rats. Micrograph shows representative c-Fos labeling in anterior insular (Al; ventral portion) and lateral orbital (LO; lateral portion) cortices for NP and P rats. Structures signaling non-persistence included $\mathrm{Al} / \mathrm{LO}$ and paraventricular thalamus (PVT). No structure in the $8 \mathrm{~d}$ group showed signaling of non-persistence. $8 \mathrm{~d}$ NP: $n=8 ; 8 d P: n=4 ; 20 \mathrm{~d}$ NP: $n=3$ (Al/LO)-4; 20d $P: n=5$. All data are shown as mean \pm SEM. ${ }^{*} p<0.05$, ${ }^{* *} p<0.01$, \#p $\leq 0.1$, ns non-significant.

exhibiting full extinction by the end of Ext-RP (20d-4d vs. 20d-10d: $p=0.03$ ) and low levels of persistent avoidance when the barrier was removed at Test (20d-4d vs. 20d-10d: $p=0.01$ ). The percentage of persistent avoidance in the $20 \mathrm{~d}-10 \mathrm{~d}$ group (37\%) matched that of the $8 \mathrm{~d}-4 \mathrm{~d}$ group $(38 \%)$, indicating that increasing the duration of Ext-RP eliminated the deleterious effects of overtraining (Fig. 5b). For c-Fos analyses, we focused on $\mathrm{AI} / \mathrm{LO}$ and $\mathrm{PL}$, two regions previously altered in $8 \mathrm{~d}-4 \mathrm{~d}$ and $20 \mathrm{~d}-4 \mathrm{~d}$ groups (see Fig. 4). Combining the two sets of overtrained rats (20d-4d, 20d-10d), we observed that the time spent on the platform (at test) was inversely correlated with activity in PL $(r=-0.45, p=0.02)$, and to a lesser extent in $\mathrm{AI} / \mathrm{LO}(r=-0.28, p=0.18)$ suggesting that activity in these areas may act to reduce persistent avoidance in overtrained (but not regularly trained) rats. Furthermore, c-Fos levels in these two areas were positively correlated across rats $(r=0.71, p<0.01)$ (Fig. 5c), consistent with the idea that inputs to PL from AI/LO may serve to reduce persistent avoidance when the barrier is removed, as previously suggested ${ }^{14}$.

\section{Discussion}

In this study, we combined conditioned active avoidance, Ext-RP and c-Fos expression to assess the effects of overtraining on persistent active avoidance. Increasing avoidance training from $8 \mathrm{~d}$ to $20 \mathrm{~d}$ did not alter expression of avoidance, but severely impaired the extinction of conditioned freezing during Ext-RP, increased anxietylike behavior, and triggered long-lasting persistence of avoidance. As for the neuronal activity profile, $8 \mathrm{~d}$ rats showing persistent avoidance also showed increased activity in regions previously associated with avoidance, as compared with non-persistent rats ${ }^{17}$. Surprisingly, there was no activation of these areas in $20 \mathrm{~d}$ rats showing persistent avoidance, as compared with the non-persistent rats. Instead, $20 \mathrm{~d}$ rats showing successful avoidance extinction displayed activation in orbital, insular and thalamic areas. Finally, extending the duration of Ext-RP training prevented the deleterious effects of overtraining. Thus, overtraining re-organizes avoidance circuits and promotes the persistence of avoidance, but this effect can be prevented by extending extinction-based training, and presumably exposure-based therapies for OCD. 


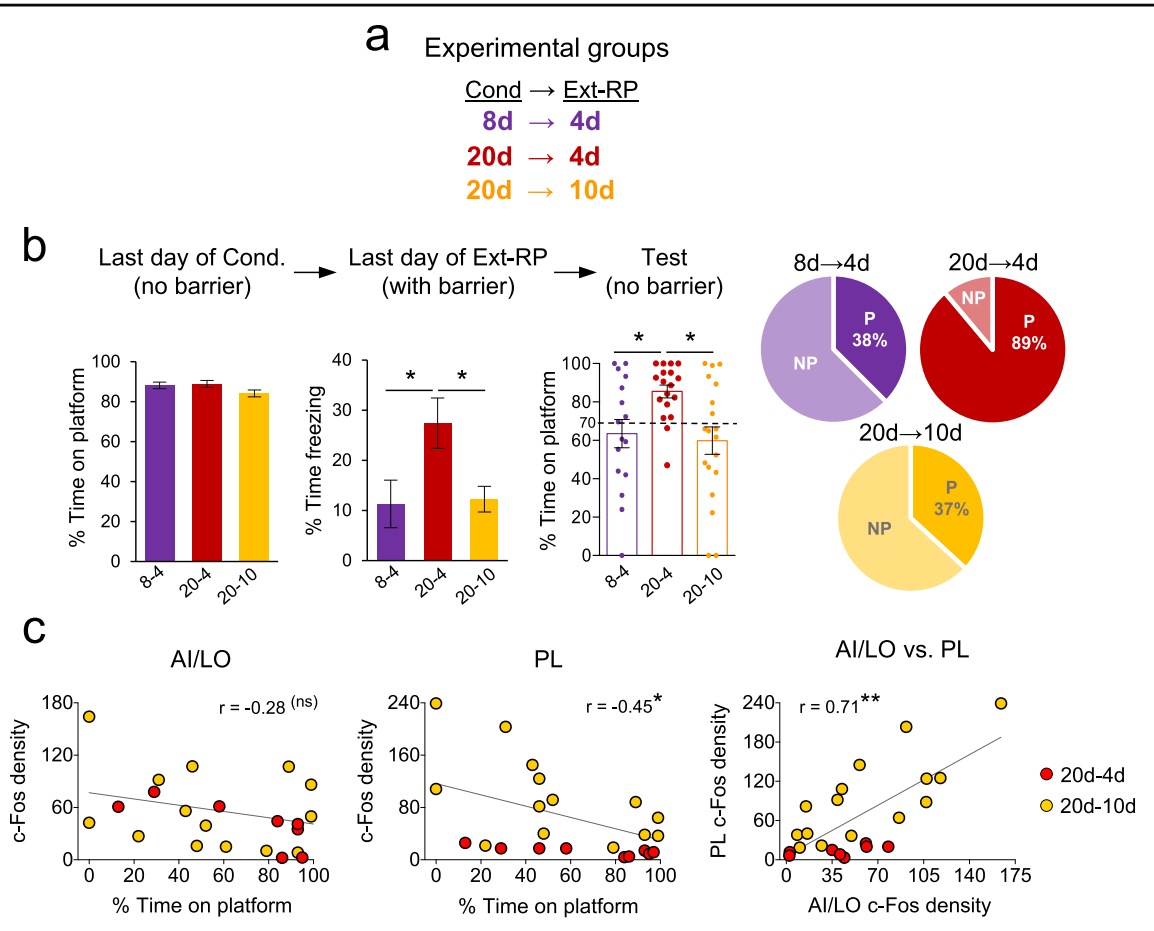

Fig. 5 Extending Ext-RP prevented the effects of overtraining. a Experimental groups receiving different durations of training for conditioning and Ext-RP phases ( $8 d-4 d, 20 d-4 d$, and 20d-10d). b While all three groups showed equivalent levels of avoidance at the end of conditioning, 20d-4d showed impaired extinction and persistent avoidance replicating our earlier findings (Fig. 2). However, extending Ext-RP (20d-10d) reduced freezing and avoidance to levels resembling the $8 \mathrm{~d}-4 \mathrm{~d}$ group. c c-Fos analysis revealed a negative correlation between time on platform at Test and AI/LO and $\mathrm{PL}$ activity in overtrained rats, as well as a positive correlation between $\mathrm{Al} / \mathrm{LO}$ and $\mathrm{PL}$ activity. This suggests that $\mathrm{Al} / \mathrm{LO}$ inputs to $\mathrm{PL}$ may serve to prevent persistent avoidance following overtraining. Behavior analyses: $8 \mathrm{~d}-4 \mathrm{~d}$ group: $n=16 ; 20 \mathrm{~d}-4 \mathrm{~d}$ group: $n=18$; $20 \mathrm{~d}-10 \mathrm{~d}$ group: $n=19$. c-Fos analyses: $20 \mathrm{~d}-4 \mathrm{~d}$ group: $n=8$ (AI/LO) $-9(\mathrm{PL}) ; 20-10$ group: $n=15$. Conditioning data shown as average of the last day of conditioning, Ext-RP data shown as a block of the first three trials of the last day of Ext-RP and Test data shown as single trial. All data are shown as mean \pm SEM. ${ }^{*} p<0.05$, ** $p<0.01$, ns non-significant.

\section{Overtraining impairs extinction of conditioned fear}

Despite similar conditioned behavior between groups, 20d rats were impaired in their ability to extinguish the tone-shock association across 4 days of Ext-RP training. Both groups showed equivalently high levels of freezing at the start of Ext-RP, but this extinguished only in the $8 \mathrm{~d}$ group. Early animal studies reported that increasing avoidance training impaired subsequent extinction, but the duration of training was much less than the $20 \mathrm{~d}$ used here. Increasing avoidance training from 2 days to 6 days (10 trials per day) delayed extinction of a "jump-up" avoidance task in rats ${ }^{26}$ and $\operatorname{dogs}{ }^{27}$, with the latter study also showing elevated anxiety in overtrained animals. These older avoidance tasks are difficult to compare with platform-mediated avoidance (PMA), for which 8 is the minimum number of days required for full conditioning ${ }^{15}$.

Another possible explanation for the extinction deficit could be that blocked access to the platform during ExtRP had an anxiogenic effect on 20d rats (but not $8 \mathrm{~d}$ rats) that interfered with extinction of conditioned fear. This is supported by the heightened anxiety exhibited by $20 \mathrm{~d}$ rats in the EPM following Ext-RP training. While some prior studies suggested that Ext-RP training facilitates subsequent extinction of avoidance ${ }^{27,28}$ others observed a deficit $^{26}$. Prior studies, however, did not examine the effects of extensive overtraining on Ext-RP. Given the larger number of conditioning trials, rats in the 20d group spent more total time on the platform during training than rats in the $8 \mathrm{~d}$ group (77 $\mathrm{min}$ vs. $28 \mathrm{~min}, t_{(76)}=52.19$, $p<0.01)$. This appears to have rendered $20 \mathrm{~d}$ rats susceptible to enhanced fear and anxiety when the platform was no longer accessible. A final possibility is that overtraining effects may be due to the passage of time between the initiation of conditioning and initiation of Ext-RP (9 days for $8 \mathrm{~d}$ group vs. 21 days for the $20 \mathrm{~d}$ group) rather than the additional training trials, as previously observed $^{29,30}$. Our current findings cannot exclude this possibility.

\section{Overtraining leads to increased avoidance following Ext-RP}

As might be expected from the impairment in Pavlovian extinction, $20 \mathrm{~d}$ rats showed more avoidance than $8 \mathrm{~d}$ rats when the barrier was removed at test. Indeed, the percentage of rats showing persistent avoidance increased 
from $37 \%$ in the $8 \mathrm{~d}$ group to $75 \%$ in the $20 \mathrm{~d}$ group, yet there was no group difference in freezing levels. Even prior to tone onset at test, $20 \mathrm{~d}$ rats expressed heightened avoidance and reduced pressing, suggesting that they may have started the test session in an anxious state. Such a state would favor avoidance, which would serve to reduce passive fear (freezing) to the tone. Thus, overtraining may exacerbate the Ext-RP-induced state of anxiety which then "requires" the expression of avoidance to tamp down fear to the pending stimulus.

In this task, it is difficult to determine whether excessive avoidance in the 20d group was goal-directed (responseoutcome) or habitual (stimulus-response). Such a determination would require avoidance to be fully devalued, similar to overfeeding in conditioned foraging tasks ${ }^{31}$ or delivering multiple shocks after removing the avoidance option $^{32}$. Impaired extinction in $20 \mathrm{~d}$ rats suggests that their avoidance was likely goal-directed, driven by a heightened state of fear and anxiety. Furthermore, the reduction in persistent avoidance after returning the barrier to a corner opposite the platform suggests that $20 \mathrm{~d}$ rats interpreted the barrier as a negative occasion setter, consistent with goal-directed motivation. If avoidance in 20d rats was goal-directed during Ext-RP, it does not preclude their avoidance from being habitual during conditioning. Indeed, because ERP induces fear/anxiety processes due to the presentation of OCD triggers ${ }^{33}$, the change in sensory cues with Ext-RP may have been sufficient to shift avoidance in $20 \mathrm{~d}$ rats from habitual back to goal-directed. Nevertheless, extensive avoidance training does not always lead to the development of habits ${ }^{2}$.

\section{Overtraining alters the avoidance circuit}

Prior studies have demonstrated that prefrontal-striatalamygdala circuitry is activated by, and necessary for, the expression of active avoidance in rodents ${ }^{17,20,34-36}$. However, the neural correlates of persistent avoidance following overtraining had not been studied. We recently reported that expression of PMA is correlated with activation of PL neurons projecting to BLA, whereas extinction of PMA is correlated with activation of IL neurons projecting to BLA and $\mathrm{VS}^{20}$. Consistent with this, we have also found that poor extinction of PMA was correlated with excessive activity in PL, BLA, and NAc, and deficient activity in $\mathrm{IL}^{17}$. However, when extinction was performed with the platform removed (similar to inserting a barrier), persistent avoidance was not associated with changes in BLA or IL.

The present study replicates this finding using a barrier to block access to the platform: persistent avoiders in the $8 \mathrm{~d}$ group showed increased activity in PL and NAc, with no changes to BLA or IL (see Table 1). Thus, increased activity in PL and NAc appear to play key roles in the expression of avoidance in rats that are not overtrained $^{15,17,34,37-39}$. Because a naïve control group was not employed in the present c-Fos experiments, we cannot directly compare activity levels to a pre-training baseline. However, comparisons were across subgroups (persistent vs non-persistent), which controls for possible differences in experimental conditions (i.e. handling, context, and shocks received).

A very different picture emerged in overtrained animals. Persistent avoidance in the $20 \mathrm{~d}$ group was not accompanied by activation of PL, NAc, or amygdala nuclei ${ }^{30}$. Thus, avoidance in the $20 \mathrm{~d}$ group does not appear to be mediated by the previously described avoidance circuit $^{35,40}$. In fact, persistent $20 \mathrm{~d}$ rats showed less activity in PL than persistent $8 \mathrm{~d}$ rats, suggesting that expression of persistent avoidance shifts through different circuits over the course of avoidance training ${ }^{31,41}$. Indeed, a negative correlation between PL activity and avoidance was observed in overtrained rats either receiving 4 or 10 days of Ext-RP. A reduction in cortico-striatal activity might also be interpreted as a reduction in goal-directed control $^{42,43}$. In fact, none of the 20 regions we examined were significantly activated in the $20 \mathrm{~d}$ rats showing persistent avoidance (see Table 1), however a lack of detectable cFos expression difference does not necessarily rule out a role for a given brain region. Nonetheless, our results suggest that overtraining may shift activity away from the prefrontal-striatal circuit controlling adaptive avoidance, perhaps to a less active "default" mode favoring avoidance-like behavior.

Interestingly, the $20 \mathrm{~d}$ rats which did not avoid at test (non-persistent) showed activation of AI/LO and PVT. The agranular insular-lateral orbital cortex (AI/LO) has been previously implicated in decision making ${ }^{44,45}$, and we previously observed that inactivation of this area induced persistent avoidance ${ }^{14}$. This suggests that activation of $\mathrm{AI} / \mathrm{LO}$ may be key for maintaining low levels of avoidance following Ext-RP (i.e. extinction transfer). Consistent with this, optogenetic or deep brain stimulation of the AI/LO projections attenuated OCD-like behavior in rodents ${ }^{46}$. The paraventricular nucleus of the thalamus (PVT) has been implicated in both appetitive and aversive behaviors ${ }^{18,47}$ which are in conflict in our task, and PVT is specifically recruited under conditions of approach/avoidance conflict ${ }^{48}$. Because these structures were never activated in $8 \mathrm{~d}$ rats, this suggests that overtraining may recruit circuits capable of overriding default behaviors under conflict conditions.

\section{Clinical relevance}

In the context of OCD, compulsions often represent anxious avoidance responses. Therefore, each time a compulsion is performed, it might relieve anxiety which would further reinforce avoidance (negative reinforcement $)^{49-51}$. This might resemble the prolonged 
conditioning in our $20 \mathrm{~d}$ rats. If anxiety drives compulsions, reducing anxiety with anxiolytic drugs, such as benzodiazepines (BZDs), might be beneficial for OCD treatment ${ }^{52}$. Whereas BZDs have been shown to interfere with context-dependent extinction of conditioned fear ${ }^{53}$, the effectiveness of BZDs in reducing persistent avoidance during exposure-with-response prevention (ERP) in humans remains untested. In humans, extensive overtraining of active avoidance appears to recapitulate core symptoms of OCD such as heightened anxiety, extinction deficits, and persistent avoidance ${ }^{54}$.

Whereas some studies found hyperactivity of the $\mathrm{AI} / \mathrm{LO}$ hub in $\mathrm{OCD}^{55}$, other found hypoactivation. OCD patients displayed reduced serotonin binding in $\mathrm{AI} / \mathrm{LO}^{56}$ and reduced cognitive flexibility correlated with lower activity in the insular region ${ }^{7}$. Indeed, insular activity is associated with avoiding aversive stimuli and adaptively guiding decision-making processes ${ }^{49}$. Furthermore, damage to the insular cortex is associated with failing to avoid the worst choice $^{57}$. Together, our data suggest that prolonged expression of avoidance may make patients less responsive to exposure-based therapies, consistent with clinical observations $^{10,12}$.

An emerging idea suggests that increasing the length of ERP therapy and/or providing an intensive/residential treatment program represents an effective management approach for severe $\mathrm{OCD}^{58-61}$. Thus, patients unresponsive to a brief/standard ERP regimen might benefit from a more intensive course of $\mathrm{ERP}^{62}$, resembling the usefulness of extending Ext-RP training in the present study. Similarly, patients applying learned therapeutic strategies in home environments may ameliorate the context-induced relapse of symptoms ${ }^{63-65}$. Extending the duration of ERP therapy may rescue non-responders, possibly by augmenting activity in brain regions that drive nonpersistence. An interesting future application of our findings would be to determine if ERP therapy could be facilitated by activating insular, orbital, or thalamic areas with transcranial magnetic or direct current stimulation $^{66-68}$.

\section{Acknowledgements}

This work was supported by NIH Conte Center P50-MH086400 and R37MH058883 to GJQ, Science Scholarship UMET and Neuro-ID 5R25NS080687-08 to MSN, and the University of Puerto Rico President's Office. Authors thank, Coraly D. Velázquez-Díaz, José E. Pérez-Torres, Héctor D. Bravo-Rivera, and Kelvin Quiñones-Laracuente for their technical assistance. We also want to thank, Christian Bravo-Rivera and Maria Diehl for their comments,

\section{Author details}

'Departments of Psychiatry and Anatomy \& Neurobiology, School of Medicine, Medical Sciences Campus, University of Puerto Rico, San Juan, PR 00936, USA. ${ }^{2}$ Department of Psychiatry and Human Behavior, Alpert Medical School of Brown University and Butler Hospital and the Providence VA Medical Center, Providence, RI 02906, USA. ${ }^{3}$ Nash family Department of Neuroscience and Friedman Brain Institute, Icahn School of Medicine at Mount Sinai, New York, NY 10029, USA
Conflict of interest

The authors declare that they have no conflict of interest.

\section{Publisher's note}

Springer Nature remains neutral with regard to jurisdictional claims in published maps and institutional affiliations.

Received: 7 August 2019 Revised: 5 June 2020 Accepted: 10 June 2020 Published online: 03 July 2020

\section{References}

1. Brown, L. A. et al. Directionality of change in obsessive compulsive disorder (OCD) and suicidal ideation over six years in a naturalistic clinical sample). J. Affect Disord. 245, 841-847 (2019).

2. de Wit, S. et al. Shifting the balance between goals and habits: five failures in experimental habit induction. J. Exp. Psychol. Gen. 147, 1043-1065 (2018).

3. Lee, M. T., Mpavaenda, D. N. \& Fineberg, N. A. Habit reversal therapy in obsessive compulsive related disorders: a systematic review of the evidence and CONSORT evaluation of randomized controlled trials. Front Behav. Neurosci. 13, 79 (2019).

4. Milad, M. R. et al. Deficits in conditioned fear extinction in obsessivecompulsive disorder and neurobiological changes in the fear circuit. JAMA Psychiatry 70, 608-618 (2013).

5. McLaughlin, N. C. et al. Extinction retention and fear renewal in a lifetime obsessive-compulsive disorder sample. Behav. Brain Res. 280, $72-77$ (2015).

6. Gillan, C. M. et al. Functional neuroimaging of avoidance habits in obsessivecompulsive disorder. Am. J. Psychiatry 172, 284-293 (2015).

7. Apergis-Schoute, A. M. et al. Neural basis of impaired safety signaling in Obsessive Compulsive Disorder. Proc. Natl. Acad. Sci. USA 114, 3216-3221 (2017).

8. Milad, M. R., Rosenbaum, B. L. \& Simon, N. M. Neuroscience of fear extinction: implications for assessment and treatment of fear-based and anxiety related disorders. Behav. Res Ther. 62, 17-23 (2014).

9. Gillan, C. M. \& Sahakian, B. J. Which is the driver, the obsessions or the compulsions, in OCD? Neuropsychopharmacology 40, 247-248 (2015).

10. Gillan, C. M. et al. The role of habit in compulsivity. Eur. Neuropsychopharmacol. 26, 828-840 (2016).

11. Baxter, L. R. Jr. et al. Caudate glucose metabolic rate changes with both drug and behavior therapy for obsessive-compulsive disorder. Arch. Gen. Psychiatry 49, 681-689 (1992).

12. Foa, E. B. et al. Randomized, placebo-controlled trial of exposure and ritual prevention, clomipramine, and their combination in the treatment of obsessive-compulsive disorder. Am. J. Psychiatry 162, 151-161 (2005).

13. Simpson, H. B. et al. A randomized, controlled trial of cognitive-behaviora therapy for augmenting pharmacotherapy in obsessive-compulsive disorder. Am. J. Psychiatry 165, 621-630 (2008).

14. Rodriguez-Romaguera, J., Greenberg, B. D., Rasmussen, S. A. \& Quirk, G. J. An avoidance-based rodent model of exposure with response prevention therapy for obsessive-compulsive disorder. Biol. Psychiatry 80, 534-540 (2016).

15. Bravo-Rivera, C. et al. Neural structures mediating expression and extinction of platform-mediated avoidance. J. Neurosci. 34, 9736-9742 (2014).

16. McGuire, J. F. et al. The role of avoidance in the phenomenology of obsessivecompulsive disorder. Compr. Psychiatry 53, 187-194 (2012).

17. Bravo-Rivera, C., Roman-Ortiz, C., Montesinos-Cartagena, M. \& Quirk, G. J. Persistent active avoidance correlates with activity in prelimbic cortex and ventral striatum. Front Behav. Neurosci. 9, 184 (2015).

18. Do-Monte, F. H. et al. Thalamic regulation of sucrose seeking during unexpected reward omission. Neuron 94, 388-400 e384 (2017).

19. Martinez-Rivera, F. J. et al. Bidirectional modulation of extinction of drug seeking by deep brain stimulation of the ventral striatum. Biol. Psychiatry $\mathbf{8 0}$, 682-690 (2016).

20. Martinez-Rivera FJ et al. Prefrontal circuits signaling active avoidance retrieval and extinction. Psychopharmacology (Berl). 236, 399-406 (2018).

21. Paxinos, G. \& Watson, C. The rat brain in stereotaxic coordinates, 6th edn. (Elsevier, Amsterdam, 2007) .

22. Bouton, M. E., Westbrook, R. F., Corcoran, K. A. \& Maren, S. Contextual and temporal modulation of extinction: behavioral and biological mechanisms. Biol. Psychiatry 60, 352-360 (2006). 
23. Trask, S., Thrailkill, E. A. \& Bouton, M. E. Occasion setting, inhibition, and the contextual control of extinction in Pavlovian and instrumental (operant) learning. Behav. Process. 137, 64-72 (2017).

24. Arch, J. J. \& Abramowitz, J. S. Exposure therapy for obsessive-compulsive disorder: an optimizing inhibitory learning approach. J. Obsess-Compuls. Rel. 6, 174-182 (2015).

25. Christianson, J. P. et al. Inhibition of fear by learned safety signals: a minisymposium review. J. Neurosci. 32, 14118-14124 (2012).

26. Mineka, $\mathrm{S}$. The effects of overtraining on flooding of jump-up and shuttlebox avoidance responses. Behav. Res. Ther. 16, 335-344 (1978).

27. Solomon, R. L. \& Wynne, L. C. Traumatic avoidance learning: the principles of anxiety conservation and partial irreversibility. Psychol. Rev. 61, 353-385 (1954).

28. Baum, M. Efficacy of response prevention (flooding) in facilitating the extinction of an avoidance response in rats: the effect of overtraining the response. Behav. Res. Ther. 6, 197-203 (1968).

29. Cain, C. K. Avoidance problems reconsidered. Curr. Opin. Behav. Sci. 26, 9-17 (2019).

30. Poremba, A. \& Gabriel, M. Amygdala neurons mediate acquisition but not maintenance of instrumental avoidance behavior in rabbits. J. Neurosci. 19, 9635-9641 (1999).

31. Balleine, B. W. \& O'Doherty, J. P. Human and rodent homologies in action control: corticostriatal determinants of goal-directed and habitual action. Neuropsychopharmacology 35, 48-69 (2010)

32. Fernando, A. et al. Free-operant avoidance behavior by rats after reinforcer revaluation using opioid agonists and D-amphetamine. J. Neurosci. 34 6286-6293 (2014)

33. Robbins, T. W., Vaghi, M. M. \& Banca, P. Obsessive-compulsive disorder: puzzles and prospects. Neuron 102, 27-47 (2019).

34. Martinez, R. C. et al. Active vs. reactive threat responding is associated with differential c-Fos expression in specific regions of amygdala and prefrontal cortex. Learn Mem. 20, 446-452 (2013).

35. LeDoux, J. E., Moscarello, J., Sears, R. \& Campese, V. The birth, death and resurrection of avoidance: a reconceptualization of a troubled paradigm. Mol. Psychiatry 22, 24-36 (2017)

36. Gabriel, M., Burhans, L. \& Kashef, A. Consideration of a unified model of amygdalar associative functions. Ann. N. Y Acad. Sci. 985, 206-217 (2003).

37. Diehl $M M$ et al. Active avoidance requires inhibitory signaling in the rodent prelimbic prefrontal cortex. Elife. 7, e34657 (2018)

38. Ramirez, F., Moscarello, J. M., LeDoux, J. E. \& Sears, R. M. Active avoidance requires a serial basal amygdala to nucleus accumbens shell circuit. J. Neurosci. 35, 3470-3477 (2015).

39. Oleson, E. B., Gentry, R. N., Chioma, V. C. \& Cheer, J. F. Subsecond dopamine release in the nucleus accumbens predicts conditioned punishment and its successful avoidance. J. Neurosci. 32, 14804-14808 (2012).

40. Darvas, M., Fadok, J. P. \& Palmiter, R. D. Requirement of dopamine signaling in the amygdala and striatum for learning and maintenance of a conditioned avoidance response. Learn Mem. 18, 136-143 (2011).

41. Shipman, M. L., Trask, S., Bouton, M. E. \& Green, J. T. Inactivation of prelimbic and infralimbic cortex respectively affects minimally-trained and extensively-trained goal-directed actions. Neurobiol. Learn Mem. 155, 164-172 (2018)

42. Hart, G. \& Balleine, B. W. Consolidation of goal-directed action depends on MAPKJERK signaling in rodent prelimbic cortex. J. Neurosci. 36, 11974-11986 (2016).

43. Hart, G., Bradfield, L. A. \& Balleine, B. W. Prefrontal corticostriatal disconnection blocks the acquisition of goal-directed action. J. Neurosci. 38, 1311-1322 (2018).

44. Daniel, M. L. et al. The anterior insula bidirectionally modulates cost-benefit decision-making on a rodent gambling task. Eur. J. Neurosci. 46, 2620-2628 (2017).
45. Jean-Richard-Dit-Bressel, P. \& McNally, G. P. Lateral, not medial, prefrontal cortex contributes to punishment and aversive instrumental learning. Learn Mem. 23, 607-617 (2016).

46. Burguiere, E., Monteiro, P., Feng, G. \& Graybiel, A. M. Optogenetic stimulation of lateral orbitofronto-striatal pathway suppresses compulsive behaviors. Science 340, 1243-1246 (2013).

47. Do-Monte, F. H., Quinones-Laracuente, K. \& Quirk, G. J. A temporal shift in the circuits mediating retrieval of fear memory. Nature 519, 460-463 (2015).

48. Choi, E. A., Jean-Richard-Dit-Bressel, P., Clifford, C. W. G., \& McNally, G. P. Paraventricular thalamus controls behavior during motivational conflict. $J$. Neurosci. 39, 4945-4958 (2019).

49. Norbury, A., Robbins, T. W., Seymour, B. Value generalization in human avoidance learning. Elife. 7, e34779 (2018)

50. Krypotos, A. M., Effting, M., Kindt, M. \& Beckers, T. Avoidance learning: a review of theoretical models and recent developments. Front Behav. Neurosci. 9, 189 (2015).

51. Vervliet, B., Lange, I. \& Milad, M. R. Temporal dynamics of relief in avoidance conditioning and fear extinction: experimental validation and clinical relevance. Behav. Res Ther. 96, 66-78 (2017).

52. Hewlett, W. A., Vinogradov, S. \& Agras, W. S. Clomipramine, clonazepam, and clonidine treatment of obsessive-compulsive disorder. J. Clin. Psychopharmacol. 12, 420-430 (1992).

53. Bouton, M. E., Kenney, F. A. \& Rosengard, C. State-dependent fear extinction with two benzodiazepine tranquilizers. Behav. Neurosci. 104, 44-55 (1990).

54. Vervliet, B. \& Indekeu, E. Low-cost avoidance behaviors are resistant to fear extinction in humans. Front Behav. Neurosci. 9, 351 (2015).

55. Luigjes, J. et al. Doubt in the insula: risk processing in obsessive-compulsive disorder. Front Hum. Neurosci. 10, 283 (2016).

56. Matsumoto, R. et al. Reduced serotonin transporter binding in the insular cortex in patients with obsessive-compulsive disorder: a [11C]DASB PET study. Neuroimage 49, 121-126 (2010).

57. Palminteri, S. et al. Critical roles for anterior insula and dorsal striatum in punishment-based avoidance learning. Neuron 76, 998-1009 (2012).

58. Leonard, R. C. et al. Residential treatment outcomes for adolescents with obsessive-compulsive disorder. Psychother. Res. 26, 727-736 (2016).

59. Taube-Schiff, M. et al. Effectiveness of intensive treatment services for obsessive compulsive disorder: outcomes from the first Canadian residential treatment program. Int J. Psychiatry Clin. Pract. 24, 59-67 (2020).

60. Bjorgvinsson, T. et al. Outcomes of specialized residential treatment for adults with obsessive-compulsive disorder. J. Psychiatr. Pract. 19, 429-437 (2013).

61. Stewart, S. E. et al. Effectiveness of intensive residential treatment (IRT) for severe, refractory obsessive-compulsive disorder. J. Psychiatr. Res. 39, 603-609 (2005).

62. Tolin, D. F., Diefenbach, G. J. \& Gilliam, C. M. Stepped care versus standard cognitive-behavioral therapy for obsessive-compulsive disorder: a preliminary study of efficacy and costs. Depress Anxiety 28, 314-323 (2011).

63. Stewart, S. E. et al. Long-term outcome following intensive residential treatment of Obsessive-Compulsive Disorder. J. Psychiatr. Res. 43, 1118-1123 (2009).

64. Veale, D. et al. Outcome of intensive cognitive behaviour therapy in a residential setting for people with severe obsessive compulsive disorder: a large open case series. Behav. Cogn. Psychother. 44, 331-346 (2016).

65. Grotte, T. et al. Three-week inpatient treatment of obsessive-compulsive disorder: a 6-month follow-up study. Front Psychol. 9, 620 (2018).

66. Brunelin, J. et al. Transcranial direct current stimulation for obsessivecompulsive disorder: a systematic review. Brain Sci. 8, 37 (2018)

67. Nauczyciel, C. et al. Repetitive transcranial magnetic stimulation over the orbitofrontal cortex for obsessive-compulsive disorder: a double-blind, crossover study. Transl. Psychiatry 4, e436 (2014).

68. Van't Wout, M. et al. Transcranial direct current stimulation may modulate extinction memory in posttraumatic stress disorder. Brain Behav. 7, e00681 (2017). 